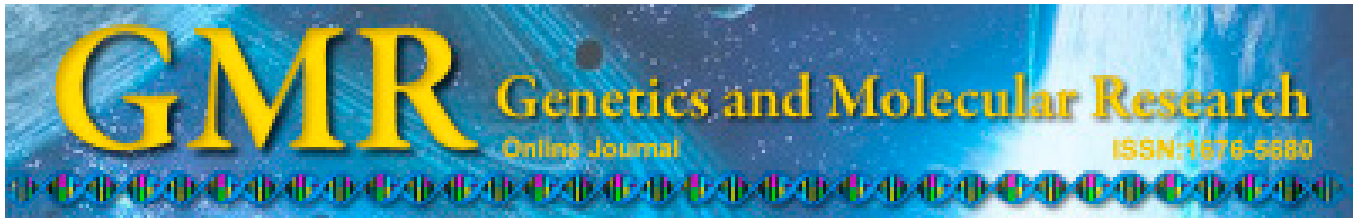

\title{
Proapoptotic effects of heme oxygenase-1 inhibitor on Kasumi-1 cells via the ATF4/ CHOP/Ire-1 $\alpha$ pathway
}

\author{
S.-X. Wei ${ }^{1,2,4,5}$, Y.-T. Wang ${ }^{6}$, Q.-X. Chai ${ }^{1,2,4}$, Q. Fang ${ }^{3}$, Y.-M. Zhang ${ }^{1,2,4}$ and \\ J.-S. Wang ${ }^{1,2,4}$ \\ ${ }^{1}$ Department of Hematology, Affiliated Hospital of Guiyang Medical College, \\ Guiyang, China \\ ${ }^{2}$ Department of Hematopoietic Stem Cell Transplantation Center of Guizhou \\ Province, Guiyang, China \\ ${ }^{3}$ Department of Pharmacy, Affiliated Hospital of Guiyang Medical College, \\ Guiyang, China \\ ${ }^{4}$ Department of Hematological Disease Diagnostic and Treat Centre of Guizhou \\ Province, Guiyang, China \\ ${ }^{5}$ Department of Clinical Biochemistry, \\ Affiliated Hospital of Guiyang Medical College, Guiyang, China \\ ${ }^{6}$ Department of Blood Transfusion, People's Hospital of Yuxi, Yuxi, China \\ Corresponding author: J.-S. Wang \\ E-mail: jishiwangen@163.com
}

Genet. Mol. Res. 14 (2): 5994-6002 (2015)

Received August 26, 2014

Accepted January 12, 2014

Published June 1, 2015

DOI http://dx.doi.org/10.4238/2015.June.1.17

ABSTRACT. We evaluated the effects of down-regulated heme oxygenase (HO)-1 expression on the proliferation of the acute myelocytic leukemia Kasumi-1 cell line by using the HO-1 inhibitor zinc protoporphyrin IX (ZnPPIX) in combination with daunorubicin (DNR), and evaluated the mechanism. The proliferation rates of cells treated with $10 \mu \mathrm{g} / \mathrm{mL}$ DNR and $10 \mu \mathrm{M}$ ZnPPIX individually or in combination for different time periods were detected using the MTT assay. The apoptotic outcomes of the blank control, ZnPPIX, DNR, and ZnPPIX groups in combination with the DNR group were detected by 
flow cytometry. The expression of $\mathrm{HO}-1$, activating transcription factor 4, CCAAT-enhancer-binding protein homologous protein, and inositolrequiring enzyme- $\alpha$ mRNA and proteins were detected by fluorescent quantitative real-time polymerase chain reaction and western blotting, respectively. Combined administration inhibited the cells most potently and time-dependently, decreased the expression of HO-1, and significantly increased the expression of activating transcription factor 4, CCAAT-enhancer-binding protein homologous protein, and inositolrequiring enzyme- $\alpha$ expression levels. The cell apoptotic rates in the blank control, DNR, ZnPPIX, and combined administration groups were $8.32 \pm 0.53,39.16 \pm 1.46,10.46 \pm 0.88$, and $56.26 \pm 2.24 \%$, respectively. Inhibiting HO-1 expression can enhance the damaging effects of DNR on Kasumi-1 cells, providing experimental evidence for the improvement of therapeutic effects on acute myelocytic leukemia in clinical practice.

Key words: Acute myelocytic leukemia; Daunorubicin; Heme oxygenase-1; Kasumi-1 cell; Zinc protoporphyrin IX

\section{INTRODUCTION}

Acute myelocytic leukemia (AML) is a malignant, clonal disorder of myeloid progenitor cells in the hematopoietic system (Rowe and Tallman, 2010) and accounts for approximately $1 \%$ of all cancer deaths. Currently, the standard induction treatment for patients consists of the antimetabolite cytarabine plus the anthracycline antibiotic daunorubicin (DNR) (Tallman et al., 2005). Despite these intensive treatment strategies, significant numbers of patients may not be effectively treated because of toxicity, drug-resistance, and severe complications related to the long-term use of chemotherapeutic agents. Therefore, it is of great significance to identify potential targets for AML treatment.

Heme oxygenase-1 (HO-1) belongs to the heat shock protein family, and thus its expression is triggered by a variety of stress-inducing stimuli, including ultraviolet irradiation, hyperthermia, inflammatory cytokines, and heavy metals (Chi et al., 2007; Agarwal and Bolisetty, 2013). HO-1 is the most interesting protein in terms of protecting different types of tumor cells from apoptosis, promoting their proliferation, and enhancing their invasion (Castilho et al., 2012; Kongpetch et al., 2012; Zhang et al., 2013; Furfaro et al., 2014). We previously reported that induction of HO-1 expression facilitated the growth of chronic myelocytic leukemia cells, whereas inhibition promoted their apoptosis (Chen et al., 2012; Wang et al., 2012). In addition, more HO-1 was expressed in AML patients than in normal donors (Ma et al., 2014), suggesting that down-regulation of HO-1 expression is a novel target for AML treatment. The regulation of HO-1 is under the control of signaling components (Shirley and Micheau, 2010; Rushworth et al., 2011) and many transcription factors, including nuclear factor-(erythroid-derived 2)-like 2-related factor 2, activating transcription factor 4 (ATF4), and activator protein-1 (He et al., 2001; Yu et al., 2014). Additionally, its expression is closely related to the endoplasmic reticulum apoptotic pathway mediated by caspases and the CCAAT-enhancer-binding protein homologous protein (CHOP) pathway (Wang et al., 2014b). 
In this study, the HO-1 inhibitor zinc protoporphyrin IX (ZnPPIX) was combined with DNR to treat AML Kasumi-1 cells to provide experimental evidence for targeted AML therapy by evaluating the effect of combined administration on cell growth; we also predicted the underlying mechanism.

\section{MATERIAL AND METHODS}

\section{Cell culture}

Kasumi-1 cells (cryopreserved in the central laboratory of Hematopoietic Stem Cell Transplantation Center of Guizhou Province, Guiyang, China) were cultured in RPMI-1640 medium (Hangzhou Tianhang Biological Technology Co., Hangzhou, China) containing 10\% fetal bovine serum (Hyclone Co., Logan, UT, USA), $100 \mathrm{U} / \mathrm{mL}$ penicillin, and $100 \mathrm{U} / \mathrm{mL}$ streptomycin at $37^{\circ} \mathrm{C}$ in a $5 \% \mathrm{CO}_{2}$ humidified atmosphere. The cells were seeded on culture plates or dishes at appropriate concentrations. This study was conducted in accordance with the Declaration of Helsinki. This study was approved by the Ethics Committee of the Hospital Affiliated to Guiyang Medical College (Guiyang, China).

\section{Methyl thiazolyl tetrazolium (MTT) assay}

After the pre-experiment, Kasumi- 1 cells were dispensed at $200 \mu \mathrm{L}$ on 96 -well plates at a density of $10 \times 10^{4}$ cells $/ \mathrm{mL}$ at $37^{\circ} \mathrm{C}$ in a $5 \% \mathrm{CO}_{2}$ humidified atmosphere. Next, $10 \mu \mathrm{g} /$ mL DNR (Sigma Co., St. Louis, MO, USA), $10 \mu \mathrm{M}$ ZnPPIX (Sigma), and $10 \mu \mathrm{M}$ ZnPPIX + $10 \mu \mathrm{g} / \mathrm{mL}$ DNR were added to the cells for 24,48 , and $72 \mathrm{~h}$. A blank control group was established, and the experiment for each group was performed in triplicate. Next, $20 \mu \mathrm{L} 5 \mathrm{mg} /$ mL MTT (Solarbio Science \& Technology Co., Ltd., Beijing, China) was added to each well, followed by incubation for $4 \mathrm{~h}$ at $37^{\circ} \mathrm{C}$. Finally, $150 \mu \mathrm{L}$ dimethyl sulfoxide was added to each well, after which absorbance was read at $570 \mathrm{~nm}$ on a microplate reader. The survival rate was calculated according to the equation: survival rate $(\%)=\mathrm{A}_{\text {drug group }} / \mathrm{A}_{\text {control group }} \times 100 \%$.

\section{Determination of apoptotic rate}

The rate of cell apoptosis was determined by flow cytometry after the cells were double-stained with annexin V-fluorescein isothiocyanate and 7-aminoactinomycin D. Briefly, $10 \times 10^{4}$ Kasumi- 1 cells were seeded on 6-well plates and incubated for $48 \mathrm{~h}$ with different treatments. Next, the cells were harvested and washed twice with ice-cold phosphate-buffered saline (10 mM HEPES, $140 \mathrm{mM} \mathrm{NaCl}$, and $\left.2.5 \mathrm{mM} \mathrm{CaCl}_{2}, \mathrm{pH}=7.4\right)$. Cell pellets were resuspended in $500 \mu \mathrm{L} 50 \mathrm{mg} / \mathrm{mL}$ 7-aminoactinomycin D and annexin-V containing RNase. After staining at room temperature for $15 \mathrm{~min}$ in the dark, the samples were then analyzed by flow cytometry using the BD FACSCalibur ${ }^{\mathrm{TM}}$ flow cytometer with BD CellQuest ${ }^{\mathrm{TM}}$ software (BD Biosciences, Franklin Lakes, NJ, USA).

\section{Real-time polymerase chain reaction (PCR) detection}

After the different treatments, total RNA was extracted using TRIzol ${ }^{\circledR}$ reagent (Invitrogen Life Technologies, Carlsbad, CA, USA). RNA was reverse-transcribed into cDNA using a reverse transcription kit (Promega Corp., Madison, WI, USA), which was stored at $-20^{\circ} \mathrm{C}$. 
Real-time PCR was performed using a real-time PCR thermocycler (Applied Biosystems, Foster City, CA, USA) and quantified using SYBR ${ }^{\circledR}$ Green PCR Master Mix (Applied Biosystems) using $1 \mu \mathrm{L}$ cDNA in a final reaction volume of $20 \mu \mathrm{L}$. The PCRs were cycled 45 times after initial denaturation $\left(94^{\circ} \mathrm{C}, 1 \mathrm{~min}\right)$ with the following parameters: denaturation at $94^{\circ} \mathrm{C}, 10 \mathrm{~s}$; annealing at $58^{\circ} \mathrm{C}, 15 \mathrm{~s}$ (ATF4, glyceraldehyde 3-phosphate dehydrogenase) or at $60^{\circ} \mathrm{C}, 10 \mathrm{~s}$ (HO-1, CHOP, Ire-1 $\alpha$ ); and extension at $72^{\circ} \mathrm{C}, 10 \mathrm{~s}$. After the PCR, the temperature was increased from 60 to $95^{\circ} \mathrm{C}$ at a rate of $2^{\circ} \mathrm{C} / \mathrm{min}$, and fluorescence was measured every $15 \mathrm{~s}$ to plot the melting curve. Glyceraldehyde 3-phosphate dehydrogenase was used as an internal control. SDS 2.2.1 software (Applied Biosystems) was used to perform relative quantification of the target genes using the $\Delta \Delta \mathrm{Ct}$ method. The primers (Takara Bio Inc., Shiga, Japan), which are shown in Table 1, were designed using the Primer Express 3.0 Software (Applied Biosystems).

Table 1. Primer sequences of gene mRNA for real-time PCR.
\begin{tabular}{lllc}
\hline Gene & Forward primer & Reverse primer & Product (bp) \\
\hline$H O-1$ & 5'-ATGGCCTCCCTGTACCACATC-3' & 5'-TGTTGCGCTCAATCTCCTCCT-3' & 55 \\
ATF4 & 5'-GTCAGTCCCTCCAACAACA-3' & 5'-GGTGTCTTCCTCCTTTATGC-3' & 37 \\
CHOP & 5'-CAGAGCTGGAACCTGAGGAG-3' & 5'-TGGATCAGTCTGGAAAAGCA-3' & 88 \\
Ire- $1 \alpha$ & 5'-GAGCAGTGGGCTCACTCTGAA-3' & 5'-GGAAATTAAAGCAACCAGGCATC-3' & 76 \\
GAPDH & 5'-GAAGGTGAAGGTCGGATGC-3' & 5'-GAAGATGGTGATGGGATTC-3' & 126 \\
\hline
\end{tabular}

\section{Western blotting}

Kasumi- 1 cells after different treatments were washed 3 times with cold phosphatebuffered saline and then lysed in Tris-buffered saline (TBS; $50 \mathrm{mM}$ Tris-HCl, $\mathrm{pH} 8.0,150 \mathrm{mM}$ $\mathrm{NaCl}, 100 \mu \mathrm{g} / \mathrm{mL}$ phenylmethylsulfonyl fluoride, and $1 \%$ Triton X-100) for $30 \mathrm{~min}$ on ice. Protein $(80 \mathrm{mg})$ was fractioned by sodium dodecyl sulfate-polyacrylamide gel electrophoresis using $10 \%$ gels and was transferred electrophoretically to hydrophilic polyvinylidene fluoride membranes (GE Healthcare Life Sciences, Piscataway, NJ, USA). The membrane was blocked with 5\% skim milk in TBS overnight and incubated with polyclonal antibodies (Cell Signaling Technology, Beverly, MA, USA) of HO-1 (1:800), ATF4 (1:800), CHOP (1:800), Ire-1 $\alpha$ $(1: 800)$ for $2 \mathrm{~h}$, washed with TBS-T (0.1\% Tween 20 in TBS), and incubated with secondary antibodies (Santa Cruz Biotechnology, Santa Cruz, CA, USA) [anti-rabbit IgG (1:1500) and anti-mouse $\operatorname{IgG}(1: 1500)$ ] at room temperature for $1 \mathrm{~h}$. The signals were visualized by exposing the membrane to X-ray film (Carestream Health, Inc., Xiamen, China), and quantified using Image J 1.44p (National Institutes of Health, Bethesda, MD, USA).

\section{Statistical analysis}

Data are reported as means \pm standard deviation. Differences between groups were analyzed using the Student $t$-test. Data analysis was performed using the SPSS16.0 software (SPSS, Inc., Chicago, IL, USA), and $\mathrm{P}<0.05$ was considered to indicate a statistically significant difference.

\section{RESULTS}

\section{MTT assay}

The survival rates of cells in the blank control group and the ZnPPIX group changed 
only minimally over an extended time, but cells in the other 2 groups (particularly the combined administration group) decreased from 24 to $72 \mathrm{~h}$ (Figure 1). Thus, DNR in combination with ZnPPIX inhibited the growth of Kasumi-1 cells in a time-dependent manner.

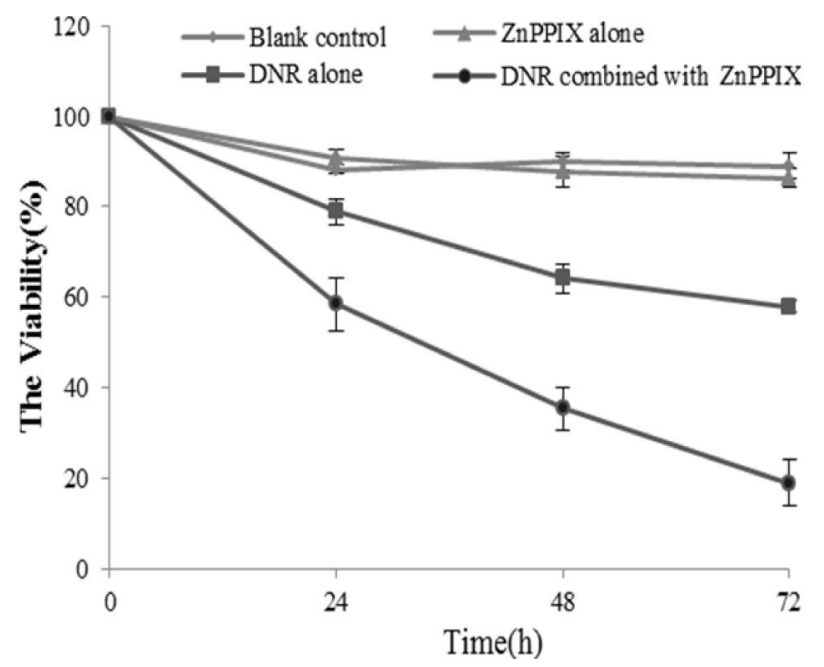

Figure 1. Time-effect curves of drug-inhibiting Kasumi-1 cell proliferation assayed using MTT.

\section{Apoptosis analysis}

Annexin-V/7-aminoactinomycin D flow cytometric staining showed that after $48 \mathrm{~h}$ of treatment, the cell apoptotic rates of the blank control, DNR, ZnPPIX, and combined administration groups were $8.32 \pm 0.53,39.16 \pm 1.46,10.46 \pm 0.88$, and $56.26 \pm 2.24 \%$, respectively. Hence, DNR exerted inhibitory effects on Kasumi-1 cells, which were significantly enhanced by the addition of ZnPPIX ( $\mathrm{P}<0.05$, Figure 2$)$.

\section{Expression of $\mathrm{HO}-1, \mathrm{ATF} 4, \mathrm{CHOP}$, and Ire-1 $\alpha$ mRNA}

Real-time PCR showed that after $48 \mathrm{~h}$ of treatment, the expression of $H O-1$ and the endoplasmic reticulum (ER) stress-related genes ATF4, CHOP, and Ire-1 $\alpha$ increased in the DNR group. In contrast, HO-1 expression was inhibited in the ZnPPIX group. Moreover, the expression levels of ATF4, CHOP, and Ire-1 $\alpha$ mRNA were highest in the combined administration group, showing statistically significant differences $(\mathrm{P}<0.05$, Figure 3$)$.

\section{Expression of HO-1, ATF4, CHOP, and Ire-1a proteins}

Western blotting showed that the expression of HO-1 protein was also remarkably inhibited in the ZnPPIX group after $48 \mathrm{~h}$ of treatment. However, DNR increased expression of the ATF4, CHOP, and Ire-1 $\alpha$ proteins. Similarly, expression levels of the ATF4, CHOP, and Ire-1 $\alpha$ proteins were highest in the combined administration group, showing statistically significant differences $(\mathrm{P}<0.05$, Figure 4$)$. 

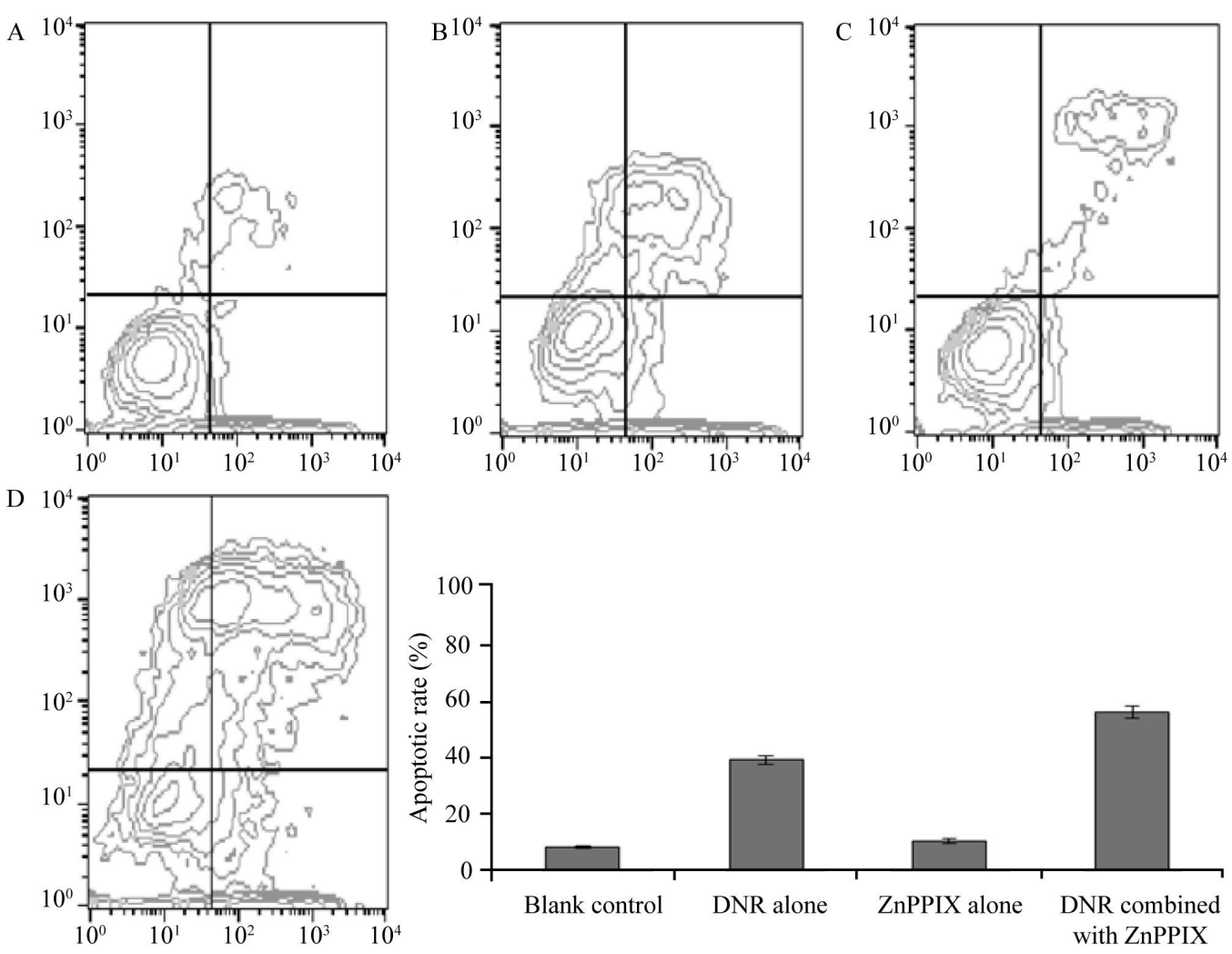

Figure 2. Apoptosis of Kasumi-1 cells detected by flow cytometry with annexin-V/7-AAD double staining. A. Control group; B. DNR group; C. ZnPPIX group; D. DNR in combination with ZnPPIX group.

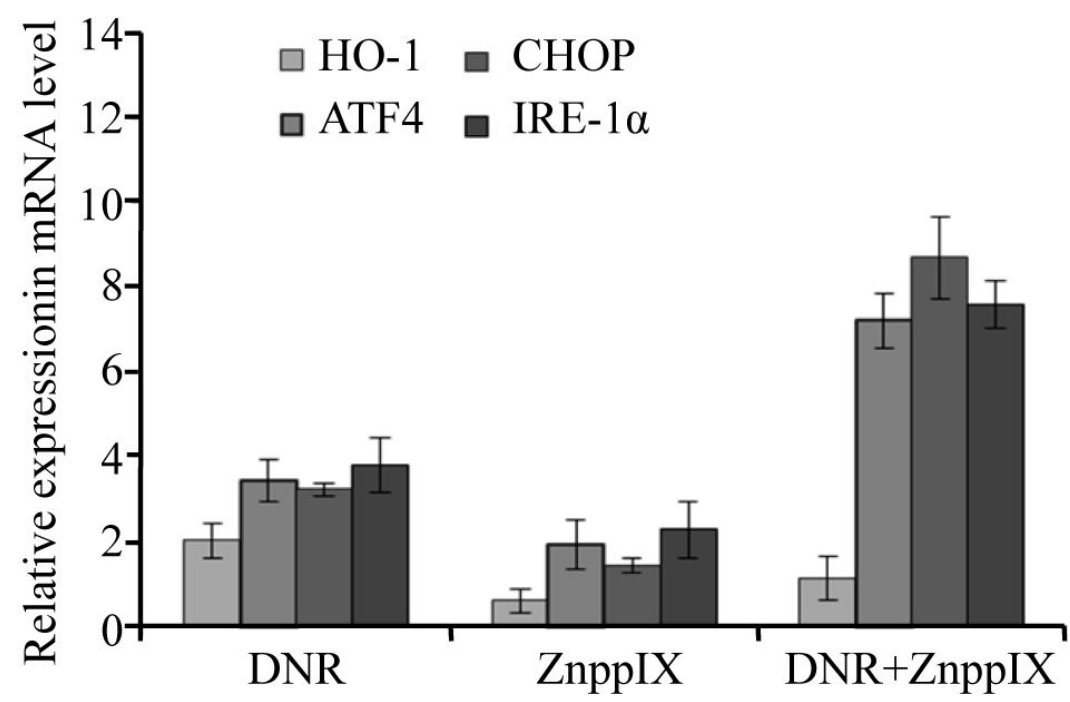

Figure 3. $H O-1, A T F 4, C H O P$, and Ire-1 $\alpha$ mRNA expression detected using real-time PCR. 


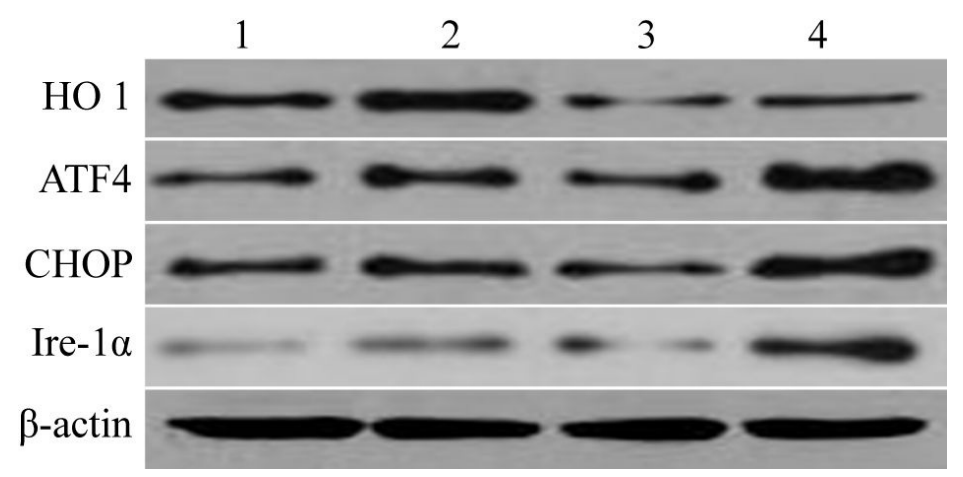

Figure 4. Protein expression of HO-1, ATF4, CHOP, and Ire-1 $\alpha$ detected by western blot. Lane 1 = control group; lane 2 = DNR group; lane $3=\mathrm{ZnPPIX}$ group; lane $4=\mathrm{DNR}$ in combination with ZnPPIX group.

\section{DISCUSSION}

AML includes a group of hematopoietic malignancies arising from abnormalities in the proliferation, differentiation, or survival of myeloid progenitors. Despite the development of several seemingly effective chemotherapy protocols for AML, a recent study in China showed that the 3-year disease-free survival rate was only $11 \%$ (Wang et al., 2005). Moreover, high-dose chemotherapy is not suitable for all patients (Hamblin, 1995). Thus, developing a safe and efficient protocol for targeted therapy has attracted global interest.

As a key enzyme for heme catabolism, HO-1 can be induced to be highly expressed by several simulating factors such as heavy metals, endotoxin, shock, and inflammatory factors. Cells generate a defensive, protective response against external environmental stimuli by inducing HO-1 expression (Wang et al., 2014a), making HO-1 a potential promoting factor for the growth of lung cancer, liver cancer, malignant glioma, and other tumor cells (Zou et al., 2011; Tertil et al., 2014). We previously reported that inducing HO-1 expression by hemin elevated the survival rate of chronic myelocytic leukemia cells, indicating that HO-1 and the survival of such cells were closely associated. The protoporphyrin derivative ZnPPIX can specifically suppress the activity of HO-1 by inhibiting the production of biliverdin and by interfering with that of $\mathrm{CO}$. Protoporphyrins are prone to aggregation in a variety of malignant tumor cells (Fang et al., 2012; Wang et al., 2013), and in vitro and in vivo administration of $\mathrm{ZnPP}$ can boost the therapeutic effects of chemotherapeutic agents. The effects of inhibited HO-1 expression on the growth of AML cells have not been widely reported. In particular, the HO-1 inhibitor has never been combined with DNR to treat AML cells. In general, ZnPPIX allows DNR to induce the apoptosis of AML cells more effectively by mitigating the simultaneous protective effects of $\mathrm{HO}-1$.

In this study, the influence of inhibited $\mathrm{HO}-1$ gene expression on the role of DNR in Kasumi-1 cell death was evaluated. The MTT assay showed that DNR in combination with ZnPPIX time-dependently suppressed the growth of Kasumi-1 cells, showing significantly lower survival rates than cells in other groups. Annexin-V/aminoactinomycin D staining also showed that combining DNR with HO-1 inhibitor ZnPPIX significantly increased the apoptosis rate of Kasumi- 1 cells compared with DNR alone. Therefore, HO-1 may be involved in the regulation of AML cell survival, as demonstrated by the promotion of cell apoptosis when HO-1 expression 
was inhibited.

The ER apoptotic pathway depends on caspases, and the ER contains a crucial cellular pool of $\mathrm{Ca}^{2+}$ ions, whose concentration changes can result in cellular stress. However, if overstressed, the failed recovery from ER stress eventually leads to cell apoptosis (Jeschke et al., 2009). Because we previously demonstrated that silencing of HO-1 expression promoted the caspase cascade activation and that intracellular $\mathrm{Ca}^{2+}$ accumulation facilitated cell apoptosis, we examined whether down-regulation of HO-1 expression could promote AML cell apoptosis via the ER stress-related ATF4/CHOP/Ire-1 $\alpha$ pathway. As detected by real-time PCR and western blotting, DNR facilitated the expression of ATF4, CHOP, and Ire- $1 \alpha$ in Kasumi-1 cells, which was augmented by combined treatment with ZnPPIX; this treatment dramatically reduced HO-1 mRNA and protein expression. Thus, inhibiting HO-1 expression contributed to Kasumi-1 cell apoptosis by activating ER stress.

In summary, HO-1 was related to the survival of AML cells, the down-regulation of which effectively promoted the apoptosis of Kasumi- 1 cells by inducing ER stress, which is consistent with our previous study showing silenced HO-1 expression with HO-1 siRNA lentivirus. Our findings provide experimental support for improved therapeutic effects on AML and for targeted AML therapy.

\section{Conflicts of interest}

The authors declare no conflict of interest.

\section{ACKNOWLEDGMENTS}

Research supported by grants from the National Natural Science Foundation of China (\#81270636 and \#81070444).

\section{REFERENCES}

Agarwal A and Bolisetty S (2013). Adaptive responses to tissue injury: role of heme oxygenase-1. Trans. Am. Clin. Climatol. Assoc. 124: 111-122.

Castilho Á, Aveleira CA, Leal EC, Simões NF, et al. (2012). Heme oxygenase-1 protects retinal endothelial cells against high glucose-and oxidative/nitrosative stress-induced toxicity. PLoS One 7: e42428.

Chen C, Wang JS, Qin D, Yang Y, et al. (2012). The effect of retrovirus-mediated HO-1 gene on chronic myeloid leukemia resistance cell K562/A02 apoptosis induced by nilotinib. Zhonghua Xue Ye Xue Za Zhi 33: 383-387.

Chi L, Ke Y, Luo C, Gozal D, et al. (2007). Depletion of reduced glutathione enhances motor neuron degeneration in vitro and in vivo. Neuroscience 144: 991-1003.

Fang J, Greish K, Qin H, Liao L, et al. (2012). HSP32 (HO-1) inhibitor, copoly (styrene-maleic acid)-zinc protoporphyrin IX, a water-soluble micelle as anticancer agent: in vitro and in vivo anticancer effect. Eur. J. Pharm. Biopharm. 81: 540-547.

Furfaro AL, Piras S, Passalacqua M, Domenicotti C, et al. (2014). HO-1 up-regulation: a key point in high-risk neuroblastoma resistance to bortezomib. Biochim. Biophys. Acta 1842: 613-622.

Hamblin TJ (1995). Disappointments in treating acute leukemia in the elderly. N. Engl. J. Med. 332: 1712-1713.

$\mathrm{He}$ CH, Gong P, Hu B, Stewart D, et al. (2001). Identification of activating transcription factor 4 (ATF4) as an Nrf2interacting protein. Implication for heme oxygenase-1 gene regulation. J. Biol. Chem. 276: 20858-20865.

Jeschke MG, Gauglitz GG, Song J, Kulp GA, et al. (2009). Calcium and ER stress mediate hepatic apoptosis after burn injury. J. Cell Mol. Med. 13: 1857-1865.

Kongpetch S, Kukongviriyapan V, Prawan A, Senggunprai L, et al. (2012). Crucial role of heme oxygenase-1 on the sensitivity of cholangiocarcinoma cells to chemotherapeutic agents. PLoS One 7: e34994.

Ma D, Fang Q, Li Y, Wang J, et al. (2014). Crucial role of heme oxygenase-1 in the sensitivity of acute myeloid leukemia 
cell line Kasumi-1 to ursolic acid. Anticancer Drugs 5: 406-414.

Rowe JN and Tallman MS (2010). How I treat acute myeloid leukemia. Blood 116: 3147-3156.

Rushworth SA, Zaitseva L, Langa S, Bowles KM, et al. (2011). FLIP regulation of HO-1 and TNF signalling in human acute myeloid leukemia provides a unique secondary anti-apoptotic mechanism. Oncotarget 1: 359-366.

Shirley S and Micheau O (2010). The heme oxygenase-1 and c-FLIP in acute myeloid leukemias: two non-redundant but mutually exclusive cellular safeguards protecting cells against TNF-induced cell death? Oncotarget 1: 317-319.

Tallman MS, Gilliland DG and Rowe JM (2005). Drug therapy for acute myeloid leukemia. Blood 106: 1154-1163.

Tertil M, Skrzypek K, Florczyk U, Wdglarczyk K, et al. (2014). Regulation and novel action of thymidine phosphorylase in non-small cell lung cancer: crosstalk with Nrf2 and HO-1. PLoS One 9: e97070.

Wang J, Hu X and Jiang H (2014a). Nrf-2-HO-1-HMGB1 axis: an important therapeutic approach for protection against myocardial ischemia and reperfusion injury. Int. J. Cardiol. 172: 223-224.

Wang JS, Yang C, Fang Q, Wei SX, et al. (2012). K562 cell line resistance to nilotinib induced in vitro and preliminary investigation of its mechanisms. Zhonghua Xue Ye Xue Za Zhi 33: 906-910.

Wang S, Avery JE, Hannafon BN, Lind SE, et al. (2013). Zinc protoporphyrin suppresses cancer cell viability through a heme oxygenase-1-independent mechanism: the involvement of the Wnt/ $\beta$-catenin signaling pathway. Biochem. Pharmacol. 85: 1611-1618.

Wang Y, Wei S, Wang J, Fang Q, et al. (2014b). Phenethyl isothiocyanate inhibits growth of human chronic myeloid leukemia K562 cells via reactive oxygen species generation and caspases. Mol. Med. Rep. 10: 543-549.

Wang YY, Zhou GB, Yin T, Chen B, et al. (2005). AML1-ETO and C-KIT mutation/overexpression in $\mathrm{t}(8 ; 21)$ leukemia: implication in stepwise leukemogenesis and response to Gleevec. Proc. Natl. Acad. Sci. U. S. A. 102: 1104-1109.

Yu JB, Shi J, Gong LR, Dong SA, et al. (2014). Role of Nrf2/ARE pathway in protective effect of electroacupuncture against endotoxic shock-induced acute lung injury in rabbits. PLoS One 9: e104924.

Zhang L, Liu YL, Chen GX, Cui B, et al. (2013). Heme oxygenase-1 promotes Caco-2 cell proliferation and migration by targeting CTNND1. Chin. Med. J. 126: 3057-3063.

Zou C, Zhang H, Li Q, Xiao H, et al. (2011). Heme oxygenase-1: a molecular brake on hepatocellular carcinoma cell migration. Carcinogenesis 32: 1840-1848. 